\title{
UM ALGORITMO DE PLANOS-DE-CORTE PARA O NÚMERO CROMÁTICO FRACIONÁRIO DE UM GRAFO
}

\author{
Manoel Campêlo* \\ Prog. de Mestrado e Doutorado em Ciência da Computação \\ e Dep. de Estatística e Matemática Aplicada \\ Universidade Federal do Ceará (UFC) \\ Fortaleza - CE, Brasil \\ mcampelo@lia.ufc.br \\ Victor A. Campos \\ Ricardo C. Corrêa \\ Prog. de Mestrado e Doutorado em Ciência da Computação \\ Universidade Federal do Ceará (UFC) \\ Fortaleza-CE, Brasil \\ campos@lia.ufc.br; correa@lia.ufc.br \\ * Corresponding author / autor para quem as correspondências devem ser encaminhadas \\ Recebido em 06/2008; aceito em 01/2009 após 1 revisão \\ Received June 2008; accepted January 2009 after one revision
}

\section{Resumo}

O número cromático fracionário $\chi_{F}(G)$ de um grafo $G$ é um conhecido limite inferior para seu número cromático $\chi(G)$. Experimentos relatados na literatura mostram que usar $\chi_{F}(G)$, em lugar do tamanho da clique máxima, pode ser muito mais eficiente para orientar a busca em um algoritmo tipo branch-and-bound para determinação de $\chi(G)$. Uma dificuldade, porém, é tratar o modelo linear conhecido para $\chi_{F}(G)$, o qual apresenta um número exponencial de variáveis e demanda um caro processo de geração de colunas. Neste trabalho, examinamos uma formulação alternativa para obter um limite inferior para $\chi_{F}(G)$ que possui um número de variáveis linear no tamanho do grafo, porém um número exponencial de restrições. Utilizamos o método de planos-de-corte para lidar com esse inconveniente. Algumas heurísticas de separação são propostas, e experimentos computacionais mostram que valores muito próximos de $\chi_{F}(G)$, em muitos casos iguais, são encontrados em tempo inferior à implementação com geração de colunas.

Palavras-chave: número cromático; planos-de-corte; otimização combinatória.

\begin{abstract}
The fractional chromatic number $\chi_{F}(G)$ of a graph $G$ is a well-known lower bound for its chromatic number $\chi(G)$. Experiments reported in the literature show that using $\chi_{F}(G)$, instead of the size of the maximum clique, can be much more efficient to drive a search in a branch-and-bound algorithm for finding $\chi(G)$. One difficulty though is to deal with the linear program that is known for $\chi_{F}(G)$. Such a formulation has an exponential number of variables and demands an expensive column generation process. In this work, we investigate the use of an alternative formulation to find a lower bound for $\chi_{F}(G)$, which has a linear number of variables but an exponential number of constraints. We use a cutting plane method to deal with this inconvenience. Some separation heuristics are proposed, and some computational experiments were carried out. They show that values very close to $\chi_{F}(G)$ (in many cases exact values) are found in less time than that required by the column generation.
\end{abstract}

Keywords: chromatic number; cutting planes; combinatorial optimization. 


\section{Introdução}

Dado um inteiro positivo $k$, uma $k$-coloração de um grafo $G=(V, E)$ é uma atribuição de valores de $\{1, \ldots, k\}$ aos vértices de $G$ tal que cada vértice receba pelo menos um valor e que vértices adjacentes recebam valores diferentes. O problema de coloração de vértices consiste em encontrar uma $\chi(G)$-coloração de $G$, sendo $\chi(G)$, conhecido como número cromático de $G$, o menor número tal que $G$ admite uma $\chi(G)$-coloração. Este problema é um dos mais estudados em teoria dos grafos pela sua relevância tanto do ponto de vista teórico, visto que até mesmo achar uma aproximação para o número cromático é um problema computacionalmente difícil (Lund \& Yannakakis, 1994), quanto do de aplicações, como escalonamento de tarefas (deWerra, 1985), alocação de frequências (Gamst, 1986), alocação de registros em compiladores (Chow \& Hennessy, 1990) e o método de elementos finitos (Saad, 1996).

A importância do problema de coloração tem incentivado a investigação de algoritmos enumerativos, orientados por limites inferiores, para o número cromático de $G$ ou de subgrafos de $G$ (Johnson \& Trick, 1996). Historicamente, as primeiras tentativas nessa direção tomavam o provável tamanho de uma clique máxima como limite inferior, como é o caso dos algoritmos tipo branch-and-bound descritos por Brélaz (1979), Brown (1972), Glover, Parker \& Ryan (1996), Kubale \& Jackowski (1985), Peemöller (1983) e Sewell (1996). Porém, como o número cromático de um grafo pode ser arbitrariamente maior do que a sua clique máxima, essa abordagem tende a examinar um número de subproblemas que somente é tolerável para grafos com poucos vértices.

Procurando reduzir o tamanho da árvore de busca, Mehrotra \& Trick (1996) utilizaram o número cromático fracionário como limite inferior para $\chi(G)$. O número cromático fracionário $\chi_{F}(G)$ é a menor razão $k / \ell$ que permite uma atribuição de $\ell$ elementos do conjunto $\{1, \ldots, k\}$ a cada vértice de $G$ de tal forma que os subconjuntos atribuídos a vértices adjacentes sejam disjuntos (Larsen, Propp \& Ullman, 1995). Sendo $S$ o conjunto de todos os conjuntos independentes maximais de $G$ e associando uma variável a cada elemento de $S$, chega-se a

$$
\chi_{F}(G)=\min _{x \in[0,1]^{|S|}} \sum_{s \in \mathcal{S}} x_{s} \text { sujeito a } \sum_{s \mid v \in S} x_{s} \geq 1, v \in V .
$$

Este programa linear é usado para calcular limites inferiores para $\chi(G)$ pelo algoritmo apresentado por Mehrotra \& Trick (1996), que se mostrou muito mais eficiente do que aqueles baseados no tamanho de uma clique maximal. No entanto, dado o número exponencial de variáveis em (1), essa abordagem encontra dificuldades, que podem ser contornadas de forma efetiva em alguns casos com um procedimento de geração de colunas também proposto por Mehrotra \& Trick (1996). Ressaltamos que determinar $\chi_{F}(G)$ é um problema NP-difícil (Lund \& Yannakakis, 1994).

Uma abordagem alternativa origina-se na formulação de programação inteira para o problema de coloração de vértices, proposta por Campêlo, Corrêa \& Frota (2004) e aprimorada por Campêlo, Campos \& Corrêa (2008), que se baseia na ideia de vértices representantes de cores. Em relação a (1), a relaxação linear dessa formulação tem a vantagem de possuir um número de variáveis e de restrições que são, respectivamente, linear e quadrático no tamanho do grafo. Em contrapartida, seu valor ótimo é, em geral, inferior a 
$\chi_{F}(G)$. Este limite inferior, porém, pode ser fortalecido pelo acréscimo de desigualdades válidas também propostas por Campêlo, Campos \& Corrêa (2008).

Neste trabalho, avaliamos uma relaxação do modelo baseado em representantes de cores na qual consideramos um número exponencial de restrições originadas de algumas das desigualdades válidas estudadas por Campêlo, Campos \& Corrêa (2008). Para lidar com o número exponencial de restrições, utilizamos o método de planos-de-corte (Dantzig, Fulkerson \& Johnson, 1954; Nemhauser \& Wolsey, 1988). Algumas heurísticas de separação são propostas, e resultados de experimentos computacionais realizados para avaliar o limite inferior obtido para $\chi_{F}(G)$ são apresentados. As heurísticas de separação propostas são adaptações de ideias que podem ser encontradas na literatura (Hoffman \& Padberg, 1993; Nemhauser \& Sigismindi, 1992). Nosso principal objeto de investigação é o seu uso na determinação do número cromático fracionário de $G$. Nesse sentido, uma comparação com os resultados obtidos por Mehrotra \& Trick (1996) mostra que valores muito próximos do número cromático fracionário, em muitos casos iguais, são encontrados em tempo inferior à implementação com geração de colunas.

O restante do texto está organizado da seguinte maneira. Na Seção 2, a notação utilizada e o programa linear empregado no algoritmo de planos-de-corte são descritos. A apresentação das heurísticas de separação é o assunto da Seção 3, enquanto os demais detalhes de implementação aparecem na Seção 4. A descrição dos experimentos computacionais e dos resultados obtidos está na Seção 5. Finalmente, a Seção 6 é dedicada a conclusões e comentários.

\section{Formalização do problema}

\subsection{Notação}

A notação adotada ao longo do texto é a seguinte. Utilizamos $u v$ para nos referir à aresta $(u, v) \in E$. O complemento de $G$ é denotado por $\bar{G}=(V, \bar{E})$ e $|\bar{E}|=\bar{m}$. A vizinhança e a antivizinhança de $u$ são dadas por $N(u)=\{v \in V \mid u v \in E\}$ e $\bar{N}(u)=V \backslash(N(u) \cup\{u\})$, respectivamente. Dada uma orientação de $G$, ou seja, uma função $\sigma: E \rightarrow V$ tal que $\sigma(u v) \in\{u, v\}$, definimos a vizinhança positiva de $u$ por $N^{+}(u)=\{v \in N(u) \mid \sigma(u v)=v\}$ e a vizinhança negativa como $N^{-}(u)=\{v \in N(u) \mid \sigma(u v)=u\}$. Se uma orientação é definida para $\bar{G}$, as antivizinhanças positiva e negativa podem ser definidas de forma similar e são denotadas por $\bar{N}^{+}(u)$ e $\bar{N}^{-}(u)$, respectivamente.

Um ciclo em $G$ é uma sequência de arestas $\left\langle u_{0} u_{1}, u_{1} u_{2}, \ldots, u_{k} u_{k+1}\right\rangle$ tal que $u_{k+1}=u_{0}$, sendo um ciclo direcionado se $\sigma\left(u_{j} u_{j+1}\right)=u_{j+1}$, para $j=1,2, \ldots, k$. Uma orientação de $G$ é acíclica se não definir ciclo direcionado.

Se $H \subseteq V$, então $G[H]=(H, E[H])$ é o subgrafo induzido em $G$ por $H$, cujos vértices são os elementos de $H$ e cujas arestas são aquelas de $G$ com ambas as extremidades em $H$. Quando $H$ é tal que $G[H]$ possui um conjunto vazio de arestas, $H$ é chamado de conjunto independente de $G$, sendo maximal se não existir outro conjunto independente em 
$G$ que o contenha. Uma clique (maximal) de $G$ ocorre quando $H$ é um conjunto independente (maximal) de $\bar{G}$. Um buraco de $G$ é a denominação usada para $H$ se o subgrafo induzido $E[H]$ define um ciclo. Diz-se que o buraco é ímpar quando sua quantidade de vértices (ou arestas) é ímpar.

\subsection{Formulação por vértices representantes}

Suponha uma ordem $\prec$ definida sobre os vértices de $G$, o que induz uma orientação acíclica $\sigma$ em $\bar{G}$ tal que, para cada $u v \in \bar{E}, \sigma(u v)=v$ se $u \prec v$. Dada essa orientação, usamos $G^{-}(v)$ para representar $G\left[\bar{N}^{-}(v)\right]$ e $G^{+}(v)$ para representar $G\left[\bar{N}^{+}(v)\right]$. Sendo acíclica, a orientação $\sigma$ produz dois conjuntos especiais não-vazios: o conjunto de vértices-fonte $S=\left\{s \in V \mid \bar{N}^{-}(s)=\varnothing\right\}$ e o conjunto de vértices-sumidouro $T=\left\{t \in V \mid \bar{N}^{+}(t)=\varnothing\right\}$.

Uma $k$-coloração de $G=(V, E), \quad k \geq \chi(G)$, pode ser descrita por um conjunto $R=\left\{r_{1}, r_{2}, \cdots, r_{k}\right\}$ de vértices e uma família $\mathbf{W}=\left\{W_{1}, \ldots, W_{k}\right\}$ de conjuntos independentes de $G$, onde cada $W_{i}$, contido em $\bar{N}^{+}\left(r_{i}\right)$, é formado pelos vértices que recebem a mesma cor de $r_{i}$. Cada vértice $r_{i}$ é chamado o representante da cor $i$, enquanto os vértices em $W_{i}$ são representados por $r_{i}$. Note que $r_{i}$ é o menor vértice na ordem recebendo a cor $i$ e, portanto, se $u$ e $v$ são vértices tais que $u \prec v$, então $v$ não pode representar a cor de $u$. Particularmente, um vértice $u \in S$ não pode ser representado por qualquer outro vértice, o que significa que $S \subseteq R$.

Para caracterizar essa descrição, definimos um vetor $x \in\{0,1\}^{\bar{m}}$ que contém variáveis binárias indexadas pelas arestas de $\bar{G}$, orientadas segundo $\prec$, tal que $x_{u v}=1$ se, e somente se, $u$ representa $v$. Por simplicidade, para todos $u \in V$ e $H \subseteq \bar{N}^{+}(u)$, adotamos a notação $x(u, H)=\sum_{v \in H} x_{u v}$. Caso $H$ contenha um único vértice $v$ ou somente as extremidades de uma única aresta $v w$, utilizamos $x(u, v)$ ou $x(u, v w)$, respectivamente. De modo similar, quando $H \subseteq \bar{N}^{-}(u)$, denotamos $x(H, u)=\sum_{v \in H} X_{v u}$. No caso em que $H=\bar{N}^{-}(u)$, adotamos a notação mais compacta

$$
\bar{x}(u)=1-x\left(\bar{N}^{-}(u), u\right) .
$$

Usando essa notação, conclui-se, em decorrência do exposto por Campêlo, Campos \& Corrêa (2008), que $x \in\{0,1\}^{\bar{m}}$ define uma coloração de $G$ se

$$
\begin{aligned}
x(u, v w) & \leq \bar{x}(u), \quad u \in V \backslash T, v w \in E\left[\bar{N}^{+}(u)\right], \\
x(u, v) & \leq \bar{x}(u), \quad u \in V \backslash T, v \in \bar{N}^{+}(u) \text { tal que } N(v) \cap \bar{N}^{+}(u)=\varnothing, \\
\bar{x}(u) & \geq 0, \quad u \in T .
\end{aligned}
$$

A condição (5) corresponde a $x\left(\bar{N}^{-}(u), u\right) \leq 1$, assegurando que $u \in T$ é representado por no máximo um vértice na sua antivizinhança negativa. Esse mesmo fato é assegurado para 
$u \in V \backslash T$ por (3), (4) e pela integralidade de $x$. Como consequência, a notação $\bar{x}(u)$ definida em (2) expressa a atribuição de um valor binário a $1-x\left(\bar{N}^{-}(u), u\right)$, o qual indica se $u$ é representado $(\bar{x}(u)=0)$ ou representante $(\bar{x}(u)=1)$. Já as desigualdades (3)-(4) garantem que as extremidades de uma aresta recebem cores diferentes e que uma cor só pode ser atribuída a um vértice se estiver representada.

Sendo assim, um vetor $x \in\{0,1\}^{\bar{m}}$ que satisfaça (3)-(5) e minimize o número de cores

$$
\sum_{u \in V} \bar{x}(u)=|V|-\sum_{u \in V \backslash S} x\left(\bar{N}^{-}(u), u\right)
$$

descreve uma coloração ótima de $G$, fornecendo pois $\chi(G)$. Naturalmente, a relaxação linear dessa formulação define um programa linear com um número polinomial de variáveis e restrições, cujo valor ótimo $\underline{\chi}(G)$ é um limite inferior para $\chi_{F}(G)$ e $\chi(G)$.

Para fortalecer esse limite, podemos considerar desigualdades válidas da forma

$$
x(u, H) \leq \alpha_{H} \bar{x}(u), \quad u \in V \backslash T,
$$

onde $H \subseteq \bar{N}^{+}(u)$ e $\alpha_{H}$ é o tamanho máximo de um conjunto independente de $G[H]$. Essas desigualdades (denominadas desigualdades externas por Campêlo, Campos \& Corrêa (2008) e rank inequalities por Rossi \& Smriglio (2001)) generalizam (3)-(4) ao estabelecer que um representante seja capaz de representar, no máximo, $\alpha_{H}$ vértices em $H$. A inclusão, na relaxação linear, de desigualdades do tipo (6) associadas a conjuntos $H$ apropriadamente escolhidos leva, conforme resultados apresentados por Campêlo, Campos \& Corrêa (2008) relacionando a formulação (1) àquela derivada de (3)-(5), a um novo limite para o número cromático fracionário dado por:

$$
\underline{\chi}_{F}(G)=\min \left\{\sum_{u \in V} \bar{x}(u) \mid(3)-(6), x \in \mathbb{R}_{+}^{\bar{m}}\right\} .
$$

Embora $\underline{\chi}_{F}(G) \geq \underline{\chi}(G)$, há que se lidar com um número elevado (potencialmente exponencial) de restrições em (7), sendo que muitas delas podem ser redundantes na descrição do poliedro correspondente.

Nas próximas seções, tratamos do problema de como obter, através do método de planos-decorte, uma aproximação $\underline{\chi}_{F}(G)$ para $\chi_{F}(G)$ derivada da inclusão de desigualdades do tipo (6) que definem facetas do poliedro associado a (3)-(5). Seguindo a descrição parcial desse poliedro apresentada por Campêlo, Campos \& Corrêa (2008), duas estruturas de $G$ são consideradas, tomando-se um conjunto $H \subseteq \bar{N}^{+}(u): H$ é uma clique maximal no subgrafo $G^{+}(u)$ ou $H$ é um buraco ímpar, maximal com relação a $\alpha_{H}$ em $G^{+}(u)$ (entende-se por maximal o fato $\alpha_{H^{\prime}}>\alpha_{H}$, para todo $H^{\prime}$ tal que $\left.H \subset H^{\prime} \subseteq \bar{N}^{+}(u)\right)$. No primeiro caso, $\alpha_{H}=1$, enquanto que no segundo $\alpha_{H}=(|H|-1) / 2$. Neste trabalho, consideramos a formulação (7) com as desigualdades (6) restritas a estes dois tipos de estruturas. 


\section{Algoritmo de planos-de-corte}

Conforme mencionado na seção anterior, adotamos o método de planos-de-corte para a obtenção de um limite inferior para $\chi_{F}(G)$, superior a $\underline{\chi}(G)$ devido à introdução de cortes do tipo (6) em (7). Nesse algoritmo, chamamos de $P L$ o programa linear que evolui com a inclusão dos planos-de-corte e denotamos por $x^{*}$ uma solução ótima do estado corrente de $P L$. Nesta seção, descrevemos as restrições que estão presentes em $P L$ desde o seu estado inicial e propomos heurísticas que determinam um vértice $u \in V \backslash T$ e um conjunto $H \subseteq \bar{N}^{+}(u)$ cuja inequação (6) correspondente seja violada por $x^{*}$. As heurísticas apresentadas correspondem aos casos em que $H$ induz uma clique maximal ou um buraco ímpar em $G^{+}(u)$.

Desigualdades do tipo (6) aparecem em diversos outros problemas que envolvem conjuntos independentes, o que tem motivado o surgimento de heurísticas de separação, particularmente para cliques maximais e buracos ímpares (Atamturk, Nemhauser \& Savelsbergh (2000), Hoffman \& Padberg (1993), Nemhauser \& Sigismindi (1992), Rossi \& Smriglio (2001)). Neste estudo acerca do problema do número cromático fracionário, damos ênfase à redução do número de estruturas enumeradas durante a separação. Este é um aspecto relevante quando comparamos a abordagem baseada em cortes, que é o foco deste trabalho, com a abordagem de geração de colunas de Mehrotra \& Trick (1996), a qual exige a enumeração das cliques maximais de $G$. Nas heurísticas que apresentamos nesta seção, as estruturas violadas são determinadas em subgrafos de $G^{+}(u)$, para cada $u \in V$, do qual retiramos vértices seguindo as propriedades estabelecidas ao longo desta seção.

\subsection{Programa linear inicial}

Para obter o $P L$ inicial, o primeiro passo é determinar uma ordem $\prec$ sobre o conjunto de vértices. Para tal, usamos a heurística QUALEX-MS (Busygin, 2002) para encontrar $S \subseteq V$ induzindo uma provável clique máxima de $G$. Em seguida, ordenamos os vértices em uma ordem não-decrescente das distâncias a $S$ em $G$. Com a ordem determinada, definimos as variáveis $x_{u v}$, com $u \in V \backslash T$ e $v \in \bar{N}^{+}(u)$.

Além das inequações do tipo $0 \leq x_{u v} \leq 1$, o $P L$ inicial inclui restrições que garantem um ponto ótimo inicial $x^{*}$ satisfazendo

$$
\bar{x}^{*}(u) \geq 0, u \in T \quad \text { e } \quad x^{*}(u, v) \leq \bar{x}^{*}(u), u \in V \backslash T, v \in \bar{N}^{+}(u) .
$$

Para $u \in T$, com $\left|\bar{N}^{-}(u)\right|>1$, colocamos em $P L$ a restrição $x\left(\bar{N}^{-}(u), u\right) \leq 1$ (o que, devido a (2), corresponde a forçar $\bar{x}(u) \geq 0)$. Já para $u \in V \backslash T$, tomamos uma partição $\mathbf{K}_{u}=\left\{K_{1}, \ldots, K_{j_{u}}\right\}$ de $\bar{N}^{+}(u)$ em cliques maximais de $G^{+}(u)$ e incluímos as restrições $x\left(u, K_{i}\right) \leq \bar{x}(u)$, para cada $i \in\left\{1, \ldots, j_{u}\right\}$, exceto quando $u \in S$ e $\left|K_{i}\right|=1$. Estando tais restrições sempre presentes em $P L$, consideramos daqui em diante que as propriedades (8) são satisfeitas. 


\subsection{Separação via cliques maximais}

Dados $u \in V$ e $H \subseteq \bar{N}^{+}(u)$ induzindo uma clique, a inequação de clique $Q_{u}(H)$ é obtida fazendo $\alpha_{H}=1$ em (6). A heurística de separação para $Q_{u}(H)$ consiste em calcular a antivizinhança reduzida de $u$, dada por

$$
\bar{N}_{1 / 2}^{+}(u)=\left\{v \in \bar{N}^{+}(u) \mid 0<x^{*}(u, v)<\bar{x}^{*}(u)\right\},
$$

efetuar a busca de cliques violadas em $G\left[\bar{N}_{1 / 2}^{+}(u)\right]$ e, finalmente, estendê-las para cliques maximais de $G^{+}(u)$.

Antes de entrarmos nos detalhes da heurística, vejamos a propriedade da antivizinhança reduzida de $u$ que nos leva a restringir a busca por cliques violadas a $G\left[\bar{N}_{1 / 2}^{+}(u)\right]$. Para isso definimos núcleo de $H$ com relação a $x^{*}$ como o subconjunto

$$
N_{u}(H)=H \cap \bar{N}_{1 / 2}^{+}(u)=\left\{v \in H \mid 0<x^{*}(u, v)<\bar{x}^{*}(u)\right\},
$$

ou seja, os vértices em $H$ que são parcialmente representados por $u$. Se a restrição (6) é violada para $H$, também o é para $N_{u}(H)$ ou para alguma aresta em $G[H]$, conforme a propriedade abaixo.

Proposição 1. A inequação $Q_{u}(H)$, para a clique $H \subseteq \bar{N}^{+}(u)$, é violada por $x^{*}$ se, $e$ somente se, $\quad x^{*}\left(u, N_{u}(H)\right)>\bar{x}^{*}(u)$ ou existe uma aresta $v w \in E[H]$ tal que $x^{*}(u, v w)>\bar{x}^{*}(u)$.

Prova: Suponha que as duas condições não sejam satisfeitas. Segue que $x^{*}(u, v) \leq x^{*}(u, v w) \leq \bar{x}^{*}(u)$, para todo $v w \in E[H]$. Se, para algum vértice $v \in H$, a equação $x^{*}(u, v)=\bar{x}^{*}(u)$ ocorre, então $x^{*}(u, w)=0$, para todo $w \in H \backslash\{v\}$. Isto implica que $x^{*}(u, H)=x^{*}(u, v)=\bar{x}^{*}(u)$, ou seja, $Q_{u}(H)$ não é violada. Por outro lado, quando $x^{*}(u, v)<\bar{x}^{*}(u)$, para todo $v \in H$, podemos escrever $H=N_{u}(H) \cup\left\{v \in H \mid x^{*}(u, v)=0\right\}$ e $\left.x^{*}(u, H)=x^{*}\left(u, N_{u}(H)\right)\right) \leq \bar{x}^{*}(u)$, novamente levando a $Q_{u}(H)$ não violada.

Caso uma das condições seja válida, claramente $Q_{u}(H)$ é violada.

A heurística de separação de cliques, apresentada no Algoritmo 1, consiste em uma enumeração parcial de cliques maximais na antivizinhança reduzida de cada $u \in V$ tal que $\bar{x}^{*}(u)>0$. Primeiro, construímos iterativamente $\bar{N}_{1 / 2}^{+}(u)$, ao mesmo tempo em que procuramos por arestas violadas. Em outras palavras, para cada $v \in \bar{N}^{+}(u)$, a ser possivelmente colocado em $\bar{N}_{1 / 2}^{+}(u)$, e cada $w \in \bar{N}^{+}(u) \cap N(v)$, verificamos se $u$ representa em mais de $\bar{X}^{*}(u)$ unidades as extremidades de $v w$. Neste caso, geramos uma clique maximal em $G^{+}(u)$ contendo $v$ e $w$. Em uma segunda etapa, tentamos garantir a inclusão de pelo 
menos uma inequação de clique para cada $v \in \bar{N}_{1 / 2}^{+}(u)$, marcando os vértices que já participaram de uma clique violada. Utilizamos o QUALEX-MS (Busygin, 2002) para gerar as cliques maximais ponderadas segundo $x^{*}$. Finalmente, é utilizada uma heurística gulosa para tornar maximais em $G^{+}(u)$ as cliques encontradas que definem cortes.

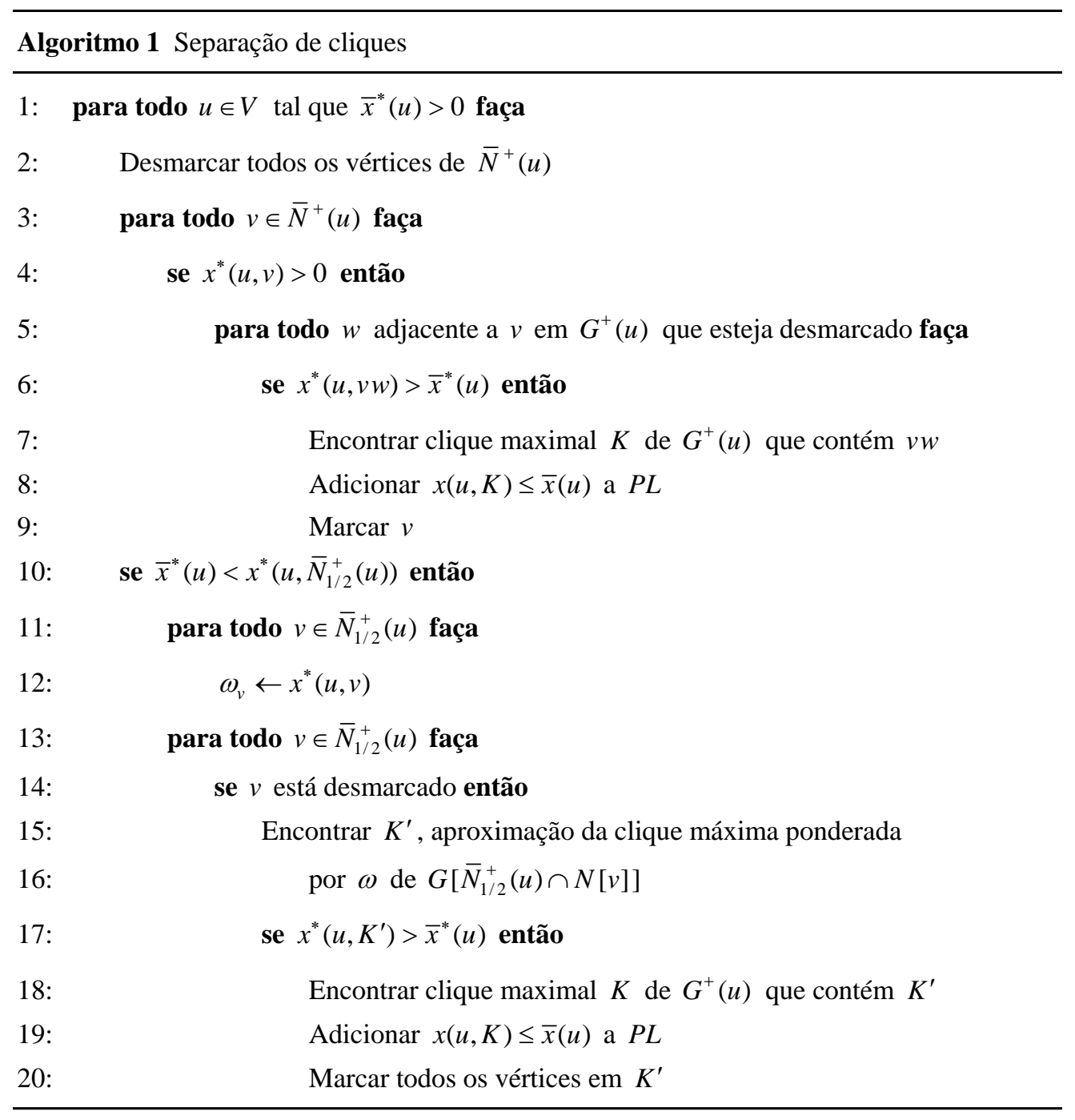

\subsection{Separação via buracos ímpares}

Nesta seção, tratamos do caso em que $H$ possui $h \geq 5$ vértices e induz um buraco ímpar em $G^{+}(u)$. A inequação de buracos $L_{u}(H)$, associada a $u$ e $H$, é obtida fazendo $\alpha_{H}=\frac{h-1}{2}$ em (6). Sua violação também se restringe à antivizinhança reduzida, como segue. 
Proposição 2. Se a inequação de buracos $L_{u}(H)$ é violada por $x^{*}$, então $H \subseteq \bar{N}_{1 / 2}^{+}(u)$ ou existe uma aresta $v w \in E[H]$ tal que $x^{*}(u, v w)>\bar{x}^{*}(u)$.

Prova: Seja $H=\left\langle v_{1}, \ldots, v_{h}\right\rangle$ um subconjunto de $\bar{N}^{+}(u)$ que induz um buraco ímpar em $G$ tal que $x^{*}(u, H)>\frac{h-1}{2} \bar{x}^{*}(u)$ e que as arestas em $E[H]$ não são violadas por representação de $u$. Por contradição, suponha que $x^{*}(u, v)=0$ ou $x^{*}(u, v)=\bar{x}^{*}(u)$, para algum $v \in H$. Sem perda de generalidade, assuma que $v=v_{1}$. Se $x^{*}\left(u, v_{1}\right)=0$, então $x^{*}(u, H)=x^{*}\left(u, P_{h-1}\right), \quad$ com $\quad P_{h-1}=\left\langle v_{2}, \ldots, v_{h}\right\rangle . \quad \mathrm{O} \quad$ somatório das inequações $x^{*}\left(u, v_{2 i} v_{2 i+1}\right) \leq \bar{x}^{*}(u)$ para $i \in\{1, \ldots,(h-1) / 2\}$ resulta em $x^{*}(u, H)=x^{*}\left(u, P_{h-1}\right) \leq \frac{h-1}{2} \bar{x}^{*}(u)$, que contradiz a hipótese. Caso $x^{*}\left(u, v_{1}\right)=\bar{x}^{*}(u)$, temos que $x^{*}\left(u, v_{2}\right)=0$, pois $x^{*}\left(u, v_{1} v_{2}\right) \leq \bar{x}^{*}(u)$. Obtemos assim a mesma contradição de antes com $v=v_{2}$. Logo, $0<x^{*}(u, v)<\bar{x}^{*}(u)$, para todo $v \in H$.

A heurística de separação proposta para buracos ímpares é baseada na seguinte propriedade, onde $P_{k}$ denota um subconjunto de $k$ vértices que induz um caminho em $G^{+}(u)$.

Proposição 3. Se $L_{u}(H)$ é violada por $x^{*}$, então, para cada $k \in\{1, \ldots, h-1\}$, existe $P_{k} \subseteq H$ tal que $x^{*}\left(u, P_{k}\right)>\frac{k}{2} \frac{h-1}{h} \bar{x}^{*}(u)$.

Prova: Seja $H=\left\langle v_{0}, \ldots, v_{h-1}\right\rangle$. Suponha que existe $k \in\{1, \ldots, h-1\}$ tal que, para todo $P_{k} \subseteq H$, a inequação $x^{*}\left(u, P_{k}\right) \leq \frac{k}{2} \frac{h-1}{h} \bar{x}^{*}(u)$ seja válida. Considere

$$
P_{k}^{i}=\left\langle v_{i}, \ldots, v_{(i+k-1) \bmod h}\right\rangle \subseteq H, \quad \text { para } i \in\{0, \ldots, h-1\} .
$$

Obtemos que $x^{*}(u, H)=\frac{1}{k} \sum_{i=0}^{h-1} x^{*}\left(u, P_{k}^{i}\right) \leq \frac{h}{k} \frac{k}{2} \frac{h-1}{h} \bar{x}^{*}(u)=\frac{h-1}{2} \bar{x}^{*}(u)$, o que contradiz a hipótese de $L_{u}(H)$ ser violada por $x^{*}$.

Pelas proposições 2 e 3 e sendo $h \geq 5$, podemos restringir a busca por inequações $L_{u}(H)$ a vértices $u \in V \operatorname{com} x^{*}(u, v)>0,4 \bar{x}^{*}(u)$, para algum $v \in \bar{N}_{1 / 2}^{+}(u)$, como feito no Algoritmo 2 . Para encontrar buraco ímpar cuja inequação correspondente é violada, procuramos nas componentes conexas $G[B]$ de $G\left[\bar{N}_{1 / 2}^{+}(u)\right]$. Esta busca enumera caminhos da forma $P_{3}=(w, v, z)$, satisfazendo a Propriedade $3 \mathrm{com} h \geq 5$, ou seja, $x^{*}\left(u, P_{3}\right)>1,2 \bar{x}^{*}(u)$, e determina um caminho mínimo entre $w$ e $z$ em $G[B \backslash(N(w) \cap N(z))]$. Uma busca em largura modificada é utilizada para gerar este caminho mínimo $P$. Note que $P$ e $u$ definem um buraco $H$ de tamanho $|P|+1$. 


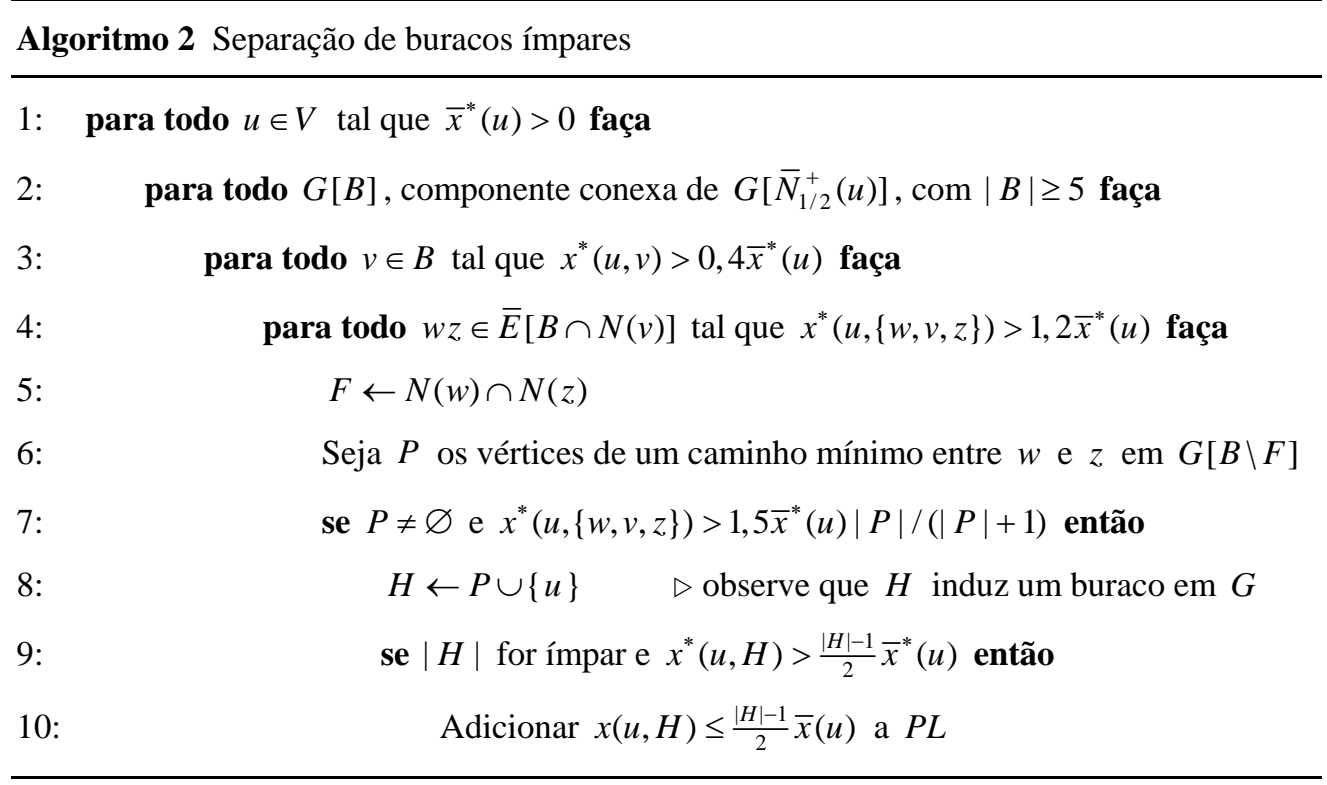

\section{Implementação}

Nesta seção, apresentamos alguns detalhes relevantes para a eficiência do algoritmo de planos-de-corte implementado.

\subsection{Pré-processamento}

A complexidade do problema pode ser substancialmente reduzida com a remoção de vértices apropriados do grafo original, como por exemplo um vértice $u \in V$ tal que $\chi_{F}(G)$ possa ser facilmente obtido a partir de $\chi_{F}(G[V \backslash\{u\}])$. A seguir, enumeramos três critérios de remoção de um vértice $u$ com tal propriedade:

1. $\bar{N}(u)=\varnothing$ : como em qualquer coloração de $G$ uma cor atribuída a $u$ deve ser distinta das cores de todos os vértices em $V \backslash\{u\}$, então $\chi_{F}(G)=\chi_{F}(G[V \backslash\{u\}])+1$.

2. $N(u) \subseteq N(v)$ para outro vértice $v$ : dada qualquer coloração fracionária ótima de $G[V \backslash\{u\}]$, uma coloração fracionária para $G$ é obtida atribuindo a $u$ as mesmas cores de $v$. Logo, $\chi_{F}(G)=\chi_{F}(G[V \backslash\{u\}]$.

3. O grau de $u$ é menor que um limitante inferior para $\omega(G)$, o tamanho da maior clique de $G$ : dado que $\omega(G) \leq \chi_{F}(G)$, no máximo $\ell(\omega(G)-1) \leq \ell\left(\chi_{F}(G)-1\right) \leq k-\ell$ cores aparecem na vizinhança de $u$ em qualquer coloração fracionária de $G[V \backslash\{u\}]$ com $k$ cores e $\ell$ cores por vértice. Assim, restam ainda $\ell$ cores para serem atribuídas a $u$, e $\chi_{F}(G)=\chi_{F}(G[V \backslash\{u\}])$. 
Após a remoção dos vértices satisfazendo as propriedades acima, o grafo pode tornar-se desconexo. O algoritmo de planos-de-corte vai trabalhar, então, sobre cada componente conexa separadamente.

\subsection{Seleção de inequações}

Conforme é usual em algoritmos de planos-de-corte, manter reduzido o número de restrições em $P L$ é essencial para limitar o tempo gasto em sua solução (Ferreira \& Wakabayashi, 1996). Em nosso caso, mantemos apenas as restrições cuja variável dual associada seja básica. Observe que esta política gera um $P L$ compacto cuja solução ótima se mantém $x^{*}$. Além disso, as restrições que definem o modelo inicial são sempre conservadas em $P L$ para que as propriedades (8) se mantenham válidas.

Uma restrição é ativa se ela está em $P L$, e inativa em caso contrário. Um repositório de restrições é utilizado para guardar algumas restrições inativas que podem eventualmente tornar-se ativas no decorrer das separações subsequentes. Particularmente, quando uma restrição é retirada de $P L$ (tornando-se inativa), ela é transferida para o repositório para que possa retornar, eventualmente, a $P L$. De modo a evitar que o tamanho do repositório se torne excessivamente grande, restrições com idade superior ou igual a 10 são descartadas. A idade de uma restrição inicia em 0 , quando ela entra no repositório, e aumenta de uma unidade a cada vez que o repositório é pesquisado, se ela permanecer inativa.

Em cada iteração, o repositório é percorrido e as heurísticas de separação são aplicadas na busca por inequações violadas por $x^{*}$, que são movidas para $P L$. Como a política de manutenção de restrições conserva apenas uma descrição compacta da solução ótima corrente, toda desigualdade encontrada que corte $x^{*}$ é adicionada a $P L$, sendo ela obtida do repositório ou por heurísticas de separação. Para muitos grafos, há a ocorrência de diversas iterações em que a quantidade de restrições adicionadas tende a ser bem grande. Mesmo assim, esta escolha mostra-se adequada para obter o maior passo possível entre os valores de duas soluções ótimas consecutivas.

\subsection{Parâmetros do algoritmo de planos-de-corte}

A forma geral de um algoritmo de planos-de-corte consiste em resolver iterativamente $P L$, adicionando cortes e removendo restrições inativas, até que nenhum corte seja adicionado ao modelo ou que sua solução seja inteira. Em nosso caso, fazemos uma modificação para que o algoritmo possa terminar mesmo com a existência de cortes sobre a solução corrente.

Definimos a margem de lucro e a tolerância como sendo, respectivamente, a melhoria necessária no limite inferior para que a iteração seja considerada boa e a quantidade de iterações que podem ocorrer consecutivamente sem que nenhuma delas seja uma iteração boa. Se a tolerância é atingida, interrompe-se o algoritmo, mesmo que ainda existam cortes a serem adicionados ao $P L$. O uso desses dois parâmetros permite parar o processo em situações nas quais o valor da função objetivo não esteja melhorando de maneira significativa com os cortes gerados durante uma sequência de iterações. Para esta implementação, baseados em testes preliminares, utilizamos a margem de lucro de $1 \%$ e a tolerância de 5 iterações. 


\section{Experimentos e resultados}

As instâncias utilizadas nos experimentos computacionais constam de um conjunto de problemas de coloração de grafos comum a diversos outros estudos experimentais (Trick, 1996). As classes de instâncias escolhidas apresentam grande diferença entre o valor da clique máxima e do número cromático fracionário ou, mesmo estes valores sendo próximos, o processo de geração de colunas mostra-se bem caro. O objetivo dos nossos experimentos é determinar a robustez do limite inferior obtido bem como o tempo necessário para calculá-lo. Para cada grafo $G$ da Tabela 1, são apresentadas as seguintes informações: número de vértices $(n)$ e arestas $(m)$, tamanho da maior clique $(\omega(G))$, melhor limite superior conhecido para $\chi(G)$ e para $\chi_{F}(G)$ (respectivamente, $\bar{\chi}(G)$ e $\bar{\chi}_{F}(G)$ ), marcado com * caso seja ótimo, além de:

- $\underline{\chi}_{F}(G)$ : limite inferior obtido para $\chi_{F}(G)$ (valor ótimo de $P L$ ao final do algoritmo),

- $\quad$ Int $=\left\lceil\underline{\chi}_{F}(G)\right\rceil:$ limite inferior obtido para $\chi(G)$,

- T: tempo total de execução em segundos,

- \%T(PL): porcentagem do tempo gasto na resolução de $P L$,

- Iter: quantidade de vezes em que $P L$ é resolvido para obter o resultado. Caso esse número seja zero, a coloração ótima foi obtida durante o pré-processamento do grafo.

Os testes foram implementados em Java, utilizando um computador Pentium IV 1,4 GHz com 1024 MBytes de memória e usando CPLEX 7.5 para resolver os problemas de programação linear. Todos os resultados, fornecidos na Tabela 1 , tiveram tempo limite de 10 minutos.

A partir destes resultados, podemos verificar a qualidade do limite inferior para $\chi(G)$ fornecido pela implementação, comparando o campo $\mathrm{Int}=\left\lceil\underline{\chi}_{F}(G)\right\rceil$ da tabela com $\left\lceil\chi_{F}(G)\right\rceil$. Observamos que nosso limite inferior difere não mais que uma unidade de $\left\lceil\chi_{F}(G)\right\rceil$ em todas as instâncias para as quais $\bar{\chi}_{F}(G)$ é conhecido, a menos de uma dessas instâncias (myciel7), onde a diferença é de duas unidades. Adicionalmente, também conseguimos determinar o número cromático fracionário exato (e sua igualdade ao número cromático) de algumas instâncias para as quais o processo de geração de colunas é excessivamente dispendioso. Isto comprova a força da formulação para um tempo de processamento relativamente baixo. 
Tabela 1 - Sumário de resultados.

\begin{tabular}{|c|c|c|c|c|c|c|c|c|c|c|}
\hline Grafo & $n$ & $M$ & $\omega(G)$ & $\bar{\chi}(G)$ & $\bar{\chi}_{F}(G)$ & $\underline{\chi}_{F}(G)$ & Int & $\mathrm{T}$ & $\% \mathrm{~T}(\mathrm{PL})$ & Iter \\
\hline mulsoli.1 & 197 & 3925 & 49 & $49 *$ & $49,00 *$ & 49,00 & 49 & $<0,1$ & 0,0 & 0 \\
\hline mulsoli.2 & 188 & 3885 & 31 & $31 *$ & $31,00 *$ & 31,00 & 31 & 2,6 & 6,3 & 1 \\
\hline mulsoli.3 & 184 & 3916 & 31 & $31 *$ & $31,00 *$ & 31,00 & 31 & 2,7 & 7,9 & 1 \\
\hline mulsoli.4 & 185 & 3946 & 31 & $31 *$ & $31,00 *$ & 31,00 & 31 & 3,4 & 6,6 & 1 \\
\hline mulsoli.5 & 186 & 3973 & 31 & $31 *$ & $31,00 *$ & 31,00 & 31 & 3,3 & 6,4 & 1 \\
\hline zeroini.1 & 211 & 4100 & 49 & $49 *$ & $49,00 *$ & 49,00 & 49 & $<0,1$ & 0,0 & 0 \\
\hline zeroini.2 & 211 & 3541 & 30 & $30 *$ & $30,00 *$ & 30,00 & 30 & 0,6 & 2,8 & 2 \\
\hline zeroini.3 & 206 & 3540 & 30 & $30 *$ & $30,00 *$ & 30,00 & 30 & 0,6 & 2,8 & 2 \\
\hline queen5.5 & 25 & 320 & 5 & $5 *$ & $5,00 *$ & 5,00 & 5 & 0,7 & 2,3 & 3 \\
\hline queen6.6 & 36 & 580 & 6 & 7* & $7,00 *$ & 6,21 & 7 & 3,8 & 9,6 & 13 \\
\hline queen7.7 & 49 & 952 & 7 & $7 *$ & $7,00 *$ & 7,00 & 7 & 3,8 & 25,4 & 6 \\
\hline queen8.8 & 64 & 1456 & 8 & $9 *$ & $8,44 *$ & 8,00 & 8 & 6,5 & 43,1 & 6 \\
\hline queen8.12 & 96 & 2736 & 12 & $12 *$ & $12,00 *$ & 12,00 & 12 & 8,2 & 41,4 & 6 \\
\hline queen9.9 & 81 & 2112 & 9 & $10 *$ & $9,00 *$ & 9,00 & 9 & 13,1 & 60,0 & 6 \\
\hline queen10.10 & 100 & 2940 & 10 & $10 *$ & - & 10,00 & 10 & 35,8 & 74,2 & 6 \\
\hline queen11.11 & 121 & 3960 & 11 & $11 *$ & $11,00^{*}$ & 11,00 & 11 & 87,7 & 84,6 & 6 \\
\hline queen12.12 & 144 & 5192 & 12 & $12^{*}$ & - & 12,00 & 12 & 208,3 & 90,7 & 6 \\
\hline queen13.13 & 169 & 6656 & 13 & $13 *$ & $13,00 *$ & 13,00 & 13 & 600,0 & 94,7 & 5 \\
\hline queen14.14 & 196 & 8372 & 14 & $14 *$ & - & 14,00 & 14 & 600,0 & 94,7 & 3 \\
\hline queen15.15 & 225 & 10360 & 15 & - & - & 15,00 & 15 & 600,0 & 93,3 & 2 \\
\hline queen16.16 & 256 & 12640 & 16 & - & - & 16,00 & 16 & 600,0 & 94,7 & 1 \\
\hline myciel3 & 11 & 20 & 2 & $4 *$ & $2,90 *$ & 2,90 & 3 & 0,6 & 1,6 & 3 \\
\hline myciel4 & 23 & 71 & 2 & $5 *$ & $3,24 *$ & 2,91 & 3 & 1,1 & 4,7 & 6 \\
\hline myciel5 & 47 & 236 & 2 & 6* & $3,55 *$ & 3,08 & 4 & 9,2 & 24,5 & 9 \\
\hline myciel6 & 95 & 755 & 2 & 7* & 3,83* & 2,99 & 3 & 86,0 & 75,4 & 8 \\
\hline myciel7 & 191 & 2360 & 2 & 8* & $4,10 *$ & 2,63 & 3 & 600,0 & 87,7 & 1 \\
\hline fullins1.3 & 30 & 100 & 3 & $4 *$ & $3,33 *$ & 3,33 & 4 & 0,9 & 6,7 & 5 \\
\hline fullins1.4 & 93 & 593 & 3 & 5 & 3,63 & 3,40 & 4 & 2,3 & 10,3 & 10 \\
\hline fullins1.5 & 282 & 3247 & 3 & 6 & 4,02 & 3,40 & 4 & 22,2 & 52,5 & 11 \\
\hline fullins 2.3 & 52 & 201 & 4 & $5 *$ & $4,25 *$ & 4,25 & 5 & 0,7 & 2,1 & 4 \\
\hline fullins 2.4 & 212 & 1621 & 4 & 6 & 4,56 & 4,26 & 5 & 2,3 & 17,4 & 6 \\
\hline fullins3.3 & 80 & 346 & 5 & 6* & $5,20 *$ & 5,20 & 6 & 0,6 & 1,7 & 2 \\
\hline fullins4.3 & 114 & 541 & 6 & $7 *$ & 6,22 & 6,17 & 7 & 0,4 & 2,6 & 1 \\
\hline fullins5.3 & 154 & 792 & 7 & 8* & 7,20 & 7,14 & 8 & 0,4 & 3,0 & 1 \\
\hline insertions1.4 & 67 & 232 & 2 & $4 *$ & 2,84 & 2,52 & 3 & 20,1 & 57,9 & 9 \\
\hline insertions1.5 & 202 & 1227 & 2 & 6 & 3,32 & 2,33 & 3 & 600,0 & 96,1 & 1 \\
\hline insertions 2.3 & 37 & 72 & 2 & $4 *$ & - & 2,34 & 3 & 2,5 & 16,8 & 10 \\
\hline insertions 2.4 & 149 & 541 & 2 & $4 *$ & 2,79 & 2,32 & 3 & 600,0 & 92,7 & 6 \\
\hline insertions3.3 & 56 & 110 & 2 & $4 *$ & - & 2,23 & 3 & 5,7 & 45,7 & 8 \\
\hline insertions3.4 & 281 & 1046 & 2 & 5 & 2,80 & - & - & 600,0 & 92,7 & - \\
\hline insertions4.3 & 79 & 156 & 2 & 3* & 2,38 & 2,18 & 3 & 13,7 & 66,8 & 8 \\
\hline
\end{tabular}




\section{Conclusões}

Neste trabalho, é investigado o uso da formulação por vértices representantes para o problema de determinação do número cromático fracionário de um grafo $G$. Nesse contexto, um algoritmo de planos-de-corte utilizando algumas heurísticas de separação para desigualdades válidas associadas a cliques maximais e buracos ímpares de subgrafos de $G$ é proposto. Experimentos computacionais realizados para avaliar o limite inferior obtido para $\chi_{F}(G)$ e, consequentemente, para $\chi(G)$, são descritos. Uma comparação com os resultados obtidos em trabalhos anteriores mostra que valores muito próximos do número cromático fracionário, em muitos casos iguais, são encontrados em tempo significativamente inferior. Uma direção para trabalhos futuros é a implementação de um algoritmo branch-and-cut para o problema de coloração de vértices usando as heurísticas propostas.

\section{Agradecimentos}

Este trabalho foi apoiado pelos projetos CNPq/Universal, CT-INFO e ALPHA. Os autores são parcialmente financiados pelo Conselho Nacional de Desenvolvimento Científico e Tecnológico - CNPq. Agradecimentos são devidos também aos revisores, cujas críticas e observações ajudaram a melhorar a qualidade do trabalho.

\section{Referências Bibliográficas}

(1) Atamturk, A.; Nemhauser, G.L. \& Savelsbergh, M.W.P. (2000). Conflict graphs in solving integer programming problems. European Journal of Operational Research, 121, 40-55.

(2) Brélaz, D. (1979). New methods to color the vertices of a graph. Communications of the ACM, 22(4), 251-256.

(3) Brown, J. (1972). Chromatic scheduling and the chromatic number problem. Management Science, 19(4), 456-463.

(4) Busygin, S. (2002). Quick almost exact maximum weight clique solver based on a generalized Motzkin-Straus formulation. <http://www.busygin.dp.ua/npc.html>.

(5) Campêlo, M.; Campos, V. \& Corrêa, R. (2008). On the asymmetric representatives formulation for the vertex coloring problem. Discrete Applied Mathematics, 156(7), 1097-1111.

(6) Campêlo, M.; Corrêa, R. \& Frota, Y. (2004). Cliques, holes and the vertex coloring polytope. Information Processing Letters, 89, 159-164.

(7) Chow, F. \& Hennessy, J. (1990). The priority-based coloring approach to register allocation. ACM Transactions on Programming Languages and Systems, 12(4), 501-536.

(8) Dantzig, G.B.; Fulkerson, D.R. \& Johnson, S.M. (1954). Solutions of a large-scale travelling salesman problem. Operations Research, 2, 393-410.

(9) de Werra, D. (1985). An introduction to timetabling. European Journal of Operations Research, 19, 151-162. 
(10) Ferreira, C.E. \& Wakabayashi, Y. (1996). Combinatória Poliédrica e Planos-de-corte Faciais. X Escola de Computação, Ed. Campinas, SP.

(11) Gamst, A. (1986). Some lower bounds for a class of frequency assignment problems. IEEE Transactions on Vehicular Technology, 35(1), 8-14.

(12) Glover, F.; Parker, M. \& Ryan, J. (1996). Coloring by tabu branch-and-bound. In: DIMACS Series in Discrete Mathematics and Theoretical Computer Science [edited by D.S. Johnson and M.A. Trick], vol. 26, 285-307.

(13) Hoffman, K. \& Padberg, M. (1993). Solving airline crew scheduling by branch and cut. Management Science, 39(6), 657-682.

(14) Johnson, D. \& Trick, M. (1996). Cliques, Coloring and Satisfiability: Second DIMACS Implementation Challenge. DIMACS Series in Discrete Mathematics and Theoretical Computer Science, vol. 26, American Mathematical Society.

(15) Kubale, M. \& Jackowski, B. (1985). A generalized implicit enumeration algorithm for graph coloring. Communications of the ACM, 28(4), 412-418.

(16) Larsen, M.; Propp, J. \& Ullman, D. (1995). The fractional chromatic number of Mycielski’s graphs. Journal of Graph Theory, 19(3), 411-416.

(17) Lund, C. \& Yannakakis, M. (1994). On the hardness of approximating minimization problems. Journal of the ACM, 41(5), 960-981.

(18) Mehrotra, A. \& Trick, M. (1996). A column generation approach for graph coloring. INFORMS Journal in Computing, 8(4), 344-354.

(19) Nemhauser, G. \& Sigismondi, G. (1992). A strong cutting plane/branch-and-bound algorithm for node packing. Journal of Operational Research Society, 43(5), 443-457.

(20) Nemhauser, G. \& Wolsey, L. (1988). Integer and Combinatorial Optimization. Wiley.

(21) Peemöller, J. (1983). A correction to Brélaz's modification of Brown's coloring algorithm. Communications of the ACM, 26(8), 595-597.

(22) Rossi, F. \& Smriglio, S. (2001). A branch-and-cut algorithm for the maximum cardinality stable set problem. Operations Research Letters, 28, 63-74.

(23) Saad, Y. (1996). Iterative Methods for Sparse Linear Systems. PWS Publishing Company, Boston, MA, USA.

(24) Sewell, E. (1996). An improved algorithm for exact graph coloring. ORSA Journal on Computing, 3, 226-373.

(25) Trick, M. (1996). Appendix: Second DIMACS Challenge test problems. In: DIMACS Series in Discrete Mathematics and Theoretical Computer Science [edited by D.S. Johnson and M.A. Trick], vol. 26, 653-657. Disponível em $<$ http://mat.gsia.cmu.edu/COLOR/instances.html $>$. 